\title{
Spectral features of voltage pulses in interacting helical channels
}

\author{
Matteo Acciai ${ }^{1,2,3, *}$, Alessio Calzona ${ }^{4}$, Matteo Carrega ${ }^{5}$, and Maura Sassetti ${ }^{2,3}$ \\ ${ }^{1}$ Dipartimento di Fisica, Università di Genova, Via Dodecaneso 33, I-16146, Genova, Italy \\ ${ }^{2}$ CNR-SPIN, Via Dodecaneso 33, I-16146, Genova, Italy \\ ${ }^{3}$ Aix-Marseille Univ, Université de Toulon, CNRS, CPT, Marseille, France \\ ${ }^{4}$ Institute for Theoretical Physics and Astrophysics, University of Würzburg, D-97074, Würzburg, Germany \\ ${ }^{5}$ NEST, Istituto Nanoscienze-CNR and Scuola Normale Superiore, Piazza San Silvestro 12, I-56127, Pisa, Italy
}

\begin{abstract}
We investigate the interplay of voltage-driven excitations and electron-electron interactions in a pair of counterpropagating helical channels capacitively coupled to a time-dependent gate. By focusing on the nonequilibrium spectral properties of the system, we show how the spectral function is modified by external drives with different time profile in presence of Coulomb interactions. In particular, we focus on a Lorentzian drive and a square single pulse. In presence of strong enough electron-electron interactions, we find that both drives can result in minimal excitations, i.e. characterized by an excess spectral function with a definite sign. This is in contrast with what happens in the non-interacting case, where only properly quantized Lorentzian pulses are able to produce minimal excitations.
\end{abstract}

\section{Introduction}

The ability to control electronic transport more and more precisely, together with the advances in nanofabrication techniques, has allowed the development of the research field known as electron quantum optics (EQO) [1, 2]. In this new framework, quantum-optic-like experiments are performed by using electrons instead of photons. The difference in particle statistics, together with the presence of the Fermi sea and possible electron-electron (e-e) interactions make EQO an interesting playground to explore new physical effects. The main ingredients of EQO are the following. Firstly, one-dimensional systems such as quantum Hall $[3,4]$ chiral edge states and two-dimensional topological insulators (2DTIs) [5-9] helical edge states can provide waveguides for electrons, thanks to their topological protection against backscattering. Secondly, singleto few-electron excitations can be generated by singleelectron sources, representing one of the major experimental breakthrough which actually allowed the development of EQO. Finally, the generated excitations can be partitioned by using quantum point contacts, playing the analog role of beamsplitters.

Although there are by now different ways of implementing single-electron sources, as reviewed in Ref. [10], two main approaches have been investigated in the context of EQO. In 2007, Fève et al. [11] developed an on-demand single-electron source based on a periodically driven quantum dot injecting electrons and holes in quantum Hall edge channels $[12,13]$. This source was subsequently employed to perform several experiments, among which it is worth

*e-mail: acciai@fisica.unige.it mentioning the fermionic analogue of the Hanbury BrownTwiss [14, 15] and Hong-Ou-Mandel [16-18] effects.

A different approach relies on the application of an external voltage drive to a one-dimensional quantum conductor. As argued by Levitov et al. [19-21], properly quantized Lorentzian pulses are able to inject into the system single electrons, devoid of spurious particle-hole pairs. This prediction has been experimentally confirmed quite recently, in 2013, and the excitations generated by quantized Lorentzian pulses have been called Levitons [22, 23]. The scientific community has shown great interest in the properties and potential applications of Levitons, as testified by the great amount of works dealing directly with this subject or related ones [24-48].

Even though the majority of EQO experiments have been performed on integer quantum Hall systems [2], proposals aiming at extending the domain of EQO to other settings have been put forward. They include the fractional quantum Hall regime [28, 29], where experimental efforts are in progress [49], superconducting systems [50, 51], and 2DTIs [52-60], a context in which the recent implementation of quantum point contacts [61] is very promising for achieving interferometric setups.

In addition, it has been shown that e-e interactions can play a relevant role and exhibit clear signatures in quantum Hall systems [18, 32, 41, 62-68]. It is well known that e-e interactions in one-dimensional systems have dramatic consequences and result in exotic phenomena, such as fractionalization [69-77] and spin-charge separation $[78,79]$. It is therefore interesting to investigate whether interaction effects can have important consequences for EQO experiments also in other frameworks, such as the helical channels emerging at the edge of 2DTIs. This is 
not interesting just from a theoretical point of view, as experimental evidence of e-e interactions in helical channels has been found in different works [80-82]. It is therefore a relevant issue to investigate how interactions affect the properties of single- to few-electron excitations generated in these systems, with particular attention to the concept of minimal excitations.

In this paper, we address this problem by considering the non-equilibrium spectral properties of a pair of interacting helical channels, coupled to an external drive, and show that they exhibit peculiar features compared to the non-interacting case. Indeed, while it is well known that properly quantized Lorentzian pulses [19-22] provide the only way to generate minimal excitations in a noninteracting one-dimensional channel, we show that this is no longer the case if interactions are present. In particular, minimal excitations featuring a spectral function with a definite sign can also be obtained with non-quantized Lorentzian pulses, as well as with other kind of drives, for instance a square pulse.

The paper is organized as follows. In Sec. 2 we present the model. In Sec. 3 we introduce the non-equilibrium spectral function and provide some general results, then specifying to the cases of Lorentzian and square pulses and discussing in which cases the excitations introduced into the system are minimal. Finally, Sec. 4 contains our conclusions. Throughout the paper we set $\hbar=1$.

\section{Model}

We consider a pair of interacting helical channels in the presence of an extended gate, located at $x<0$ and capacitively coupled to the system, as sketched in Fig. 1. The Hamiltonian

$$
H_{0}=\sum_{\sigma=\uparrow, \downarrow} \int_{-\infty}^{+\infty} d x \Psi_{\sigma}^{\dagger}(x)\left(-i v_{F} \vartheta_{\sigma} \partial_{x}\right) \Psi_{\sigma}(x)
$$

describes right-moving, spin-up electrons and leftmoving, spin-down ones. Thus, the direction of propagation is linked to the spin projection, a property known as spin-momentum locking $[5,7,8]$. In Eq. (1), $\Psi_{\sigma}^{\dagger}(x)$ is the operator creating an electron with spin $\sigma$ at position $x$, $v_{F}$ is the Fermi velocity and $\vartheta_{\uparrow / \downarrow}= \pm 1$.

Short-range density-density interactions are taken into account by the Hamiltonian

$$
H_{\mathrm{int}}=\int_{-\infty}^{+\infty} d x\left[\sum_{\sigma} \frac{g_{4}}{2}\left[n_{\sigma}(x)\right]^{2}+g_{2} n_{\uparrow}(x) n_{\downarrow}(x)\right]
$$

where $n_{\sigma}(x)=\Psi_{\sigma}^{\dagger}(x) \Psi_{\sigma}(x)$ is the density operator for electrons with spin $\sigma$ and coupling constants $g_{2}$ and $g_{4}$ refer to inter- and intra-channel interactions, respectively. Finally, the Hamiltonian describing the external drive $V(t)$ applied in the region $x<0$ is

$$
H_{\text {gate }}=-e \int_{-\infty}^{+\infty} d x \Theta(-x) V(t)\left[n_{\uparrow}(x)+n_{\downarrow}(x)\right],
$$

where $\Theta(x)$ is the Heaviside step function and $-e$ is the electron charge. The drive function $V(t)$ is assumed to be

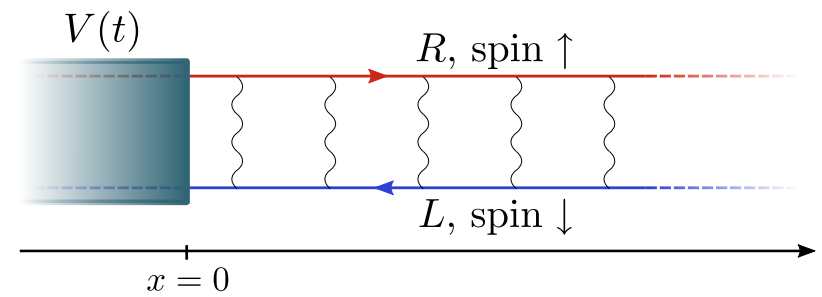

Figure 1. Schematic representation of the system considered. A pair of counterpropagating helical channels is coupled to a voltage drive $V(t)$ applied in the region $x<0$. Wiggly lines represent interactions in the system.

a single pulse with a typical time extension $\bar{w}$ and such that $\lim _{|t| \rightarrow+\infty} V(t)=0$. We notice that the single pulse $V(t)$ can be seen as a periodic drive in the limit of infinite period and it therefore features a similar phenomenology. Here, we focus on the single pulse limit which allows a more straightforward description of the low frequency behavior of the spectral function, as we discuss in Section 3.1. Notice also that the drive couples to the total electron density $[56,58]$ as the two channels associated with different spin projections are not spatially separated.

It is useful to tackle the problem by relying on the bosonization technique, which allows to express fermionic operators as $[83,84]$

$$
\Psi_{\sigma}(x)=\frac{F_{\sigma}}{\sqrt{2 \pi a}} e^{i \vartheta_{\sigma} k_{F} x} e^{-i \sqrt{2 \pi} \Phi_{\sigma}(x)},
$$

where $a$ is a short-distance cutoff, $F_{\sigma}$ is a Klein factor, $k_{F}$ the Fermi momentum and $\Phi_{\sigma}$ is a bosonic operator satisfying the commutation relations $2\left[\Phi_{\sigma}(x), \Phi_{\sigma^{\prime}}\left(x^{\prime}\right)\right]=$ $i \vartheta_{\sigma} \delta_{\sigma, \sigma^{\prime}} \operatorname{sgn}\left(x-x^{\prime}\right)$. In bosonized form, the Hamiltonian of the helical channels is diagonalized as

$$
H_{\text {edge }}=H_{0}+H_{\text {int }}=\frac{u}{2} \sum_{\eta= \pm} \int_{-\infty}^{+\infty} d x\left[\partial_{x} \Phi_{\eta}(x)\right]^{2},
$$

where $u=(2 \pi)^{-1} \sqrt{\left(2 \pi v_{F}+g_{4}\right)^{2}+g_{2}^{2}}$ is the renormalized velocity. For a Galilean invariant system, $g_{2}=g_{4}$ and thus $u=v_{F} / K[83,85,86]$ (see Eq. (7) below). Here, however, we do not make this assumption. The new fields $\Phi_{\eta}$ which diagonalize the Hamiltonian are related to $\Phi_{\sigma}$ by the transformation

$$
\Phi_{\sigma}(x)=\sum_{\eta= \pm} A_{\eta \vartheta_{\sigma}} \Phi_{\eta}(x), \quad A_{ \pm}=\frac{1}{2}\left(\frac{1}{\sqrt{K}} \pm \sqrt{K}\right)
$$

where

$$
K=\sqrt{\frac{2 \pi v_{F}-g_{2}+g_{4}}{2 \pi v_{F}+g_{2}+g_{4}}}
$$

is known as the Luttinger parameter $[83,84]$ and quantifies the interaction strength. In the non-interacting case $K=1$ and $\Phi_{ \pm}=\Phi_{\uparrow / \downarrow}$, while $K<1$ describes repulsive interactions.

By solving the equations of motion associated to the Hamiltonian $H_{\text {edge }}+H_{\text {gate }}$, one can show that the voltage 
pulse $V(t)$ simply modifies the time evolution of bosonic chiral fields as [56, 87]

$$
\Phi_{\eta}(x, t)=\phi_{\eta}(x-\eta u t, 0)+\varphi_{\eta}(x, t)
$$

where $\phi_{\eta}(x-\eta u t, 0)$ denotes the chiral time evolution with respect to $H_{\text {edge }}$ only [see Eq. (5)] and

$$
\varphi_{\eta}(x, t)=\sqrt{2 \pi K} \int_{-\infty}^{t} d t^{\prime}\left[\frac{-e}{2 \pi} V\left(t^{\prime}\right)\right] \Theta\left(\eta u t-x-\eta u t^{\prime}\right) .
$$

As far as the particle density is concerned, the voltage pulse generates excitations propagating both to the right $(x>0)$ and to the left $(x<0)$ with respect to the injection point $x=0$. These excitations retain the temporal profile $V(t)$ of the drive, apart from interaction-dependent prefactors which renormalize the charge they carry with them [87]. Explicitly, the electron density variation (with respect to its equilibrium value) $\Delta n(x, t)$ reads

$$
\Delta n(x, t)=\sum_{\eta= \pm} \partial_{x} \varphi_{\eta}(x, t)=-e \sum_{\eta= \pm} \frac{\eta K}{2 \pi u} V\left(t-\frac{\eta x}{u}\right) \Theta(\eta x) .
$$

Here, we will only consider the excitations propagating to the right, namely those in the region to the right of the one where the voltage pulse is applied (see Fig. 1). Thus, from now on we set $x>0$.

The total injected charge (in units of $-e$ ) which moves to the right is $q K$, where

$$
q=\int_{-\infty}^{+\infty} d t^{\prime} \frac{-e}{2 \pi} V\left(t^{\prime}\right)
$$

as it can be easily verified by integrating over space the excess electron density associated with right-moving excitations, i.e. considering only the term with $\eta=+$ in Eq. (10). In the presence of interactions, $\Phi_{+}$is a linear combination of fields $\Phi_{\uparrow}$ and $\Phi_{\downarrow}$ [see Eq. (6)] and thus the charge $q K$ is carried by both a spin-up packet and a spin-down one, with associated charges

$$
Q_{\uparrow, \downarrow}= \pm \sqrt{K} A_{ \pm} q .
$$

When $K=1$, we get $Q_{\uparrow}=q$ and $Q_{\downarrow}=0$ [see Eq. (6)]. This is because in this case a right-moving spin-down excitation does not exist, due to the fact that in a non-interacting helical system right-moving excitations can only have spin-up component.

\section{Non-equilibrium spectral function}

As we have mentioned, the spatial or temporal profile of the excitations generated by the drive retains the shape of $V(t)$ and thus it does not provide any information about their energy content. In order to investigate this point, we consider the non-equilibrium spectral properties $[83,88,89]$ of the system. Among all, these properties are of interest because of their connection with local probe measurements, such as tunneling conductance [90, 91]. Moreover, spectral properties are also useful to characterize minimal excitations, as we explain later in this Section.
Therefore, we consider the local spectral function, defined as

$$
\mathcal{H}_{\sigma}^{<}(\omega)=\frac{-i}{2 \pi} \int_{-\infty}^{+\infty} d \tau d t e^{i \omega \tau} G_{\sigma}^{<}(t, \tau, x) .
$$

Here, $G_{\sigma}^{<}$is the lesser local Green function given by

$$
G_{\sigma}^{<}\left(t_{1}, t_{2} ; x\right)=i\left\langle\Psi_{\sigma}^{\dagger}\left(x, t_{2}\right) \Psi_{\sigma}\left(x, t_{1}\right)\right\rangle .
$$

The variables $t$ and $\tau$ appearing in the Eq. (13), denote the average time $t=\left(t_{1}+t_{2}\right) / 2$ and the time difference $\tau=t_{1}-t_{2}$. Indeed, because of the voltage pulse which induces a non-equilibrium state, the Green functions $G_{\sigma}^{<}$ depend on both the difference $t$ and $\tau$ and not just on the latter.

To focus on the effects induced by the voltage drive only, we consider the variation of $\mathcal{H}_{\sigma}^{<}(\omega)$ with respect to equilibrium, given by

$$
\Delta \mathcal{A}_{\sigma}^{<}(\omega)=\frac{-i}{2 \pi} \int_{-\infty}^{+\infty} d \tau d t e^{i \omega \tau} \Delta G_{\sigma}^{<}(t, \tau, x),
$$

where

$\Delta G_{\sigma}^{<}\left(t_{1}, t_{2} ; x\right)=i\left\langle\Psi_{\sigma}^{\dagger}\left(x, t_{2}\right) \Psi_{\sigma}\left(x, t_{1}\right)\right\rangle-i\left\langle\psi_{\sigma}^{\dagger}\left(x, t_{2}\right) \psi_{\sigma}\left(x, t_{1}\right)\right\rangle$.

Here, $\psi_{\sigma}(x, t)$ represents the time evolution of the fermionic fields at equilibrium, without the voltage pulse [92].

By exploiting Eqs. (4), (6), (8) and (9), the variation of the lesser Green function can be expressed as [87]

$$
\Delta G_{\sigma}^{<}(t, \tau ; x)=G_{0}^{<}(\tau) P_{\sigma}(\tau) \Upsilon_{\sigma}(t, \tau, x),
$$

where

$$
G_{0}^{<}(\tau)=i\left\langle\psi_{\sigma}^{\dagger}\left(x, t_{2}\right) \psi_{\sigma}\left(x, t_{1}\right)\right\rangle=\frac{i}{2 \pi(a-i u \tau)}\left(\frac{a}{a-i u \tau}\right)^{2 A_{-}^{2}}
$$

is the well-known zero-temperature equilibrium correlator $[83,84]$,

$$
P_{\sigma}(\tau)=\frac{i u \tau-a \vartheta_{\sigma}}{i u \tau}
$$

stems from the point splitting procedure and ensures that

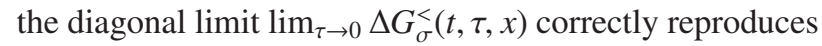
the variation of the electron density [87, 93]. The function $\Upsilon_{\sigma}(t, \tau, x)$ encodes all the effect of the voltage pulse and reads

$$
\Upsilon_{\sigma}(t, \tau, x)=\exp \left[2 \pi i \sqrt{K} A_{\vartheta_{\sigma}} \int_{t-x / u+\tau / 2}^{t-x / u-\tau / 2} \frac{-e}{2 \pi} V\left(t^{\prime}\right) d t^{\prime}\right]-1 .
$$

The (excess) lesser spectral function can be readily obtained from Eq. (17) by Fourier transform with respect to $\tau$ and integrating over $t$

$$
\begin{aligned}
\Delta \mathcal{A}_{\sigma}^{<}(\omega)= & \frac{-i}{2 \pi} \int_{-\infty}^{+\infty} d \tau d t e^{i \omega \tau} \Delta G_{\sigma}^{<}(t, \tau, x) \\
= & \frac{-i}{2 \pi} \int_{-\infty}^{+\infty} d \tau e^{i \omega \tau} G_{0}^{<}(\tau) P_{\sigma}(\tau) \\
& \times \int_{-\infty}^{+\infty} d t \Upsilon_{\sigma}(t, \tau, x)
\end{aligned}
$$


Notice that the spectral function has no spatial dependence, because the function $\Upsilon_{\sigma}$, contained in $\Delta G_{\sigma}^{<}$, depends on $t$ and $x$ only via $t-x / u$ [see Eq. (20)] and thus any dependence on $x$ is lost when performing the integration over $t$.

\subsection{General properties}

Before discussing results for specific voltage pulses, it is worth to comment on some general properties of $\Delta \mathcal{A}_{\sigma}^{<}(\omega)$. First of all, it is easy to check the sum rule

$$
\int_{-\infty}^{+\infty} d \omega \Delta \mathcal{A}_{\sigma}^{<}(\omega)=\lim _{\tau \rightarrow 0} \frac{i \vartheta_{\sigma}}{2 \pi u \tau} \int_{-\infty}^{+\infty} d t \Upsilon_{\sigma}(t, \tau, x)=\frac{Q_{\sigma}}{u}
$$

This allows us to introduce the notion of minimality also in the interacting case. In particular, one can define an excitation to be minimal if $\Delta \mathcal{A}_{\sigma}^{<}(\omega)$ has a definite sign, which obviously has to be the same as the one of $Q_{\sigma}$ [87]. Indeed, the variation with respect to equilibrium described by $\Delta \mathcal{A}_{\sigma}^{<}(\omega)$ is globally larger if the excess spectral function has in some regions a different sign compared to $Q_{\sigma}$. This can be simply understood since those regions then have to be compensated in such a way that the sum rule (22) is fulfilled. Importantly, in the non-interacting case, where a single-particle description holds, this notion of minimality corresponds to the usual requirement of not having particle-hole excitations.

Moreover, it is interesting to analytically study the low-energy behavior of $\Delta \mathcal{H}_{\sigma}^{<}(\omega)$. To this end, we have to consider the large- $\tau$ limit of Eq. (20). In particular, we safely assume the voltage pulse $V\left(t^{\prime}\right)$ to be centered around $t^{\prime}=0$, with a typical time width $\bar{w}$ (i.e. such that $V\left(t^{\prime}\right) \simeq 0$ for $\left.\left|t^{\prime}\right|>\bar{w}\right)$. In the limit $|\tau| \gg \bar{w}$ we can thus approximate

$$
\int_{t+\tau / 2}^{t-\tau / 2} \frac{-e}{2 \pi} V\left(t^{\prime}\right) d t^{\prime} \sim \begin{cases}0 & |t| \gg|\tau| / 2 \\ -q \operatorname{sgn}(\tau) & |t| \ll|\tau| / 2 \\ b(t, \tau) & |t|-|\tau| \leq \bar{w}\end{cases}
$$

where $|b(t, \tau)|<q$ is some finite function depending on the specific voltage pulse, which we will specify later. As a consequence

$$
\int_{-\infty}^{+\infty} d t \Upsilon_{\sigma}(t, \tau, x) \sim|\tau| e^{-2 \pi i q \sqrt{K} A_{\vartheta_{\sigma}} \operatorname{sgn}(\tau)}-1
$$

With this approximation we can evaluate the remaining integral over $\tau$. Indeed, for $A_{-}^{2}<1 / 2$, one finds

$$
\int_{0}^{+\infty} d \tau \cos (\omega \tau)\left(\frac{a}{u|\tau|}\right)^{2 A_{-}^{2}}=\left(\frac{a|\omega|}{u}\right)^{2 A_{-}^{2}} \frac{\Gamma\left(1-2 A_{-}^{2}\right)}{|\omega|} \sin \left(\pi A_{-}^{2}\right)
$$$$
\int_{0}^{+\infty} d \tau \sin (\omega \tau)\left(\frac{a}{u|\tau|}\right)^{2 A_{-}^{2}}=\left(\frac{a|\omega|}{u}\right)^{2 A_{-}^{2}} \frac{\Gamma\left(1-2 A_{-}^{2}\right)}{\omega} \cos \left(\pi A_{-}^{2}\right)
$$

Notice that the constraint $A_{-}^{2}<1 / 2$ means $K>0.27$, so that also quite strong interactions can be considered within

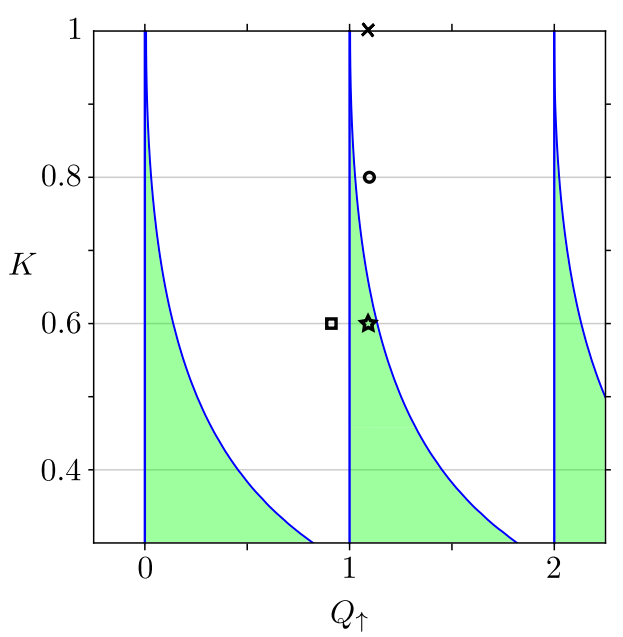

Figure 2. Sign of $D_{\uparrow}^{-}\left(Q_{\uparrow}, K\right)$ as a function of the charge $Q_{\uparrow}$ on the spin-up channel and of the interaction strength $K$. Green (white) regions correspond to $D_{\uparrow}^{-}>0\left(D_{\uparrow}^{-}<0\right)$. The blue lines correspond to $D_{\uparrow}^{-}=0$. The black markers show the parameters chosen in Figs. 3 and 4. Green regions show the parameters values for which it is possible to have minimal excitations, i.e. those featuring a spectral function with definite sign.

our results. The previous identities, together with Eq. (24), eventually give

$$
\begin{aligned}
& \lim _{\omega \rightarrow 0} \Delta \mathcal{H}_{\sigma}^{<}(\omega)=\frac{1}{|\omega|^{1-2 A_{-}^{2}}}\left[\frac{1}{2 \pi^{2}} \frac{1}{u}\left(\frac{a}{u}\right)^{2 A_{-}^{2}} \Gamma\left(1-2 A_{-}^{2}\right)\right] \\
& \times \begin{cases}D_{\sigma}^{+}\left(Q_{\sigma}\right) & \omega \rightarrow 0^{+} \\
D_{\sigma}^{-}\left(Q_{\sigma}, K\right) & \omega \rightarrow 0^{-}\end{cases}
\end{aligned}
$$

with

$$
\begin{aligned}
D_{\sigma}^{+}\left(Q_{\sigma}\right) & =1-\cos \left(2 \pi \vartheta_{\sigma} Q_{\sigma}\right) \geq 0 \\
D_{\sigma}^{-}\left(Q_{\sigma}, K\right) & =2 \sin \left(\pi \vartheta_{\sigma} Q_{\sigma}\right) \sin \left(\pi \frac{(1-K)^{2}}{2 K}-\pi \vartheta_{\sigma} Q_{\sigma}\right) .
\end{aligned}
$$

Eq. (27) describes a power-law divergence $|\omega|^{2 A_{-}^{2}-1}$ of the spectral function for $\omega \rightarrow 0^{ \pm}$. We stress that, if one of the coefficients $D_{\sigma}^{ \pm}=0$, the present analysis fails in describing the behavior of the spectral function, which could either feature a weaker divergence or not diverge at all for $\omega \rightarrow$ $0^{ \pm}$. While $D_{\sigma}^{+}=0 \Longleftrightarrow Q_{\sigma} \in \mathbb{Z}$, the condition for $D_{\sigma}^{-}$ to vanish is represented by the blue lines in Fig. 2. The divergence described by Eq. (27) for non-integer charges can be understood as a manifestation of the orthogonality catastrophe [19, 94-96].

By comparing Eq. (22), (28) and (29), it is possible to derive a general and necessary condition for $\Delta \mathcal{A}_{\sigma}^{<}(\omega)$ to have definite sign. Indeed, at least one of the following condition has to be satisfied. Either (i) the charge on the channel is integer $Q_{\sigma} \in \mathbb{Z}$, which implies that both $D_{\sigma}^{ \pm}=0$, or (ii) the charge on the channel is positive $Q_{\sigma}>0$ and the interaction strength $K$ is such that $D_{\sigma}^{-} \geq 0$. Fig. 2 highlights in green the regions in parameter space $\left(Q_{\sigma}, K\right)$ which fulfills condition (ii). Interestingly, these regions 
exist only in the presence of interactions $(K<1)$. The blue lines delimiting green regions in Fig. 2 correspond to $D_{\sigma}^{-}=0$.

In the following we will discuss some examples where the excess lesser spectral function has a definite sign, showing that interactions can make this happen for different shape of the time-dependent driving, in sharp contrast with non-interacting systems. Our examples show that, in some specific cases, the necessary condition for minimality derived before can also be sufficient. In order to keep the discussion simple and brief, we illustrate this effect by focusing on the spin-up excitation. Thus, from now on we consider $\sigma=\uparrow$.

\subsection{Lorentzian pulses}

We now illustrate our results for specific shapes of the drive $V(t)$. The first example we consider is provided by Lorentzian pulses. We recall that this is of special interest because of its property of being associated with minimal excitations and producing the minimal noise in noninteracting systems [20-23]. We now show how this simple picture is modified by the presence of interactions.

The shape of a single Lorentzian voltage pulse is described as

$$
V(t)=\frac{q}{-e} \frac{2 w}{w^{2}+t^{2}} .
$$

The full width at half maximum is given by $2 w$ and the normalization is such that Eq. (11) holds:

$$
q=\int_{-\infty}^{+\infty} d t^{\prime} \frac{-e}{2 \pi} V\left(t^{\prime}\right) .
$$

We study the lesser excess spectral function $\Delta \mathcal{A}_{\uparrow}^{<}(\omega)$ for two different interaction strengths $K=\{1,0.6\}$ and charges $Q_{\uparrow}=\{-1,0.9,1,1.1\}$. Results are summarized in Fig. 3, where minimal distributions with definite sign are highlighted in red. The first four plots (A,B,C and D) correspond to integer Levitons, i.e. $Q_{\uparrow} \in \mathbb{Z}$. As it is known [19-22, 87], in these conditions the excess spectral function $\Delta \mathcal{A}_{\uparrow}^{<}(\omega)$ has a definite sign, both in the noninteracting (panels $\mathrm{A}$ and $\mathrm{C}$ ) and in the interacting case (panels B and D). In addition, the spectral functions do not feature any divergence around $\omega \rightarrow 0$, in agreement with the condition $D_{\uparrow}^{ \pm}=0$ obtained from Eqs. (28) and (29).

In passing, we also note that the distributions for $Q_{\uparrow}=$ -1 (plots $\mathrm{C}$ and $\mathrm{D}$ ) are proportional to $\Theta(-\omega)$, both in the absence and in the presence of interactions. This feature, which has been extensively discussed in Ref. [87] in the case of a periodic drive, is peculiar of Lorentzian pulses and leads to a vanishing excess partitioning noise at a quantum point contact.

The other four plots (E,F,G and $\mathrm{H}$ ) correspond to the injection of non-integer charges and represent one of the main new results of this work. Remarkably, we show that the excess spectral function can be minimal even in this case, provided that the necessary condition (ii) is fulfilled. This means that, in the presence of interactions, a Lorentzian pulse generating an excitation which carries a non-integer charge does not necessarily result in a nonminimal spectral function. This is nicely testified by plot F, whose corresponding parameters $\left(Q_{\uparrow}=1.1, K=0.6\right)$ fall in one of the green regions of Fig. 2 (black star). By contrast, the remaining three plots (E, G and $\mathrm{H}$ ) are not minimal as they do not meet neither the first condition $\left(Q_{\uparrow}\right.$ is not integer), nor the second one (see the corresponding black markers in Fig. 2). We observe that all of these four plots feature divergences for $\omega \rightarrow 0$ which are in agreement with Eqs. (28) and (29) for $D_{\uparrow}^{ \pm}$.

\subsection{Square pulses}

As we have seen in the previous Section, due to interactions it is possible to have a spectral function with a definite sign also for excitations carrying non-integer charges. We now investigate if it is possible to achieve this situation with a different shape of the voltage drive. As an example, we show in this Section what happens in the case of square pulses. This is another standard signal and it has been considered in the non-interacting case in comparison with Lorentzian pulses [22, 23, 97]. In the absence of interactions, it produces non-minimal excitations; here, on the contrary, the presence of interactions results in a spectral function with a definite sign, as we now discuss.

We consider the following single-pulse drive:

$$
V(t)=\frac{2 q}{-e w} \Theta\left(t+\frac{\pi w}{2}\right) \Theta\left(-t+\frac{\pi w}{2}\right) .
$$

The full width of the square pulse is $\pi w$ and it is normalized in such a way that Eq. (11) holds

$$
q=\int_{-\infty}^{+\infty} d t^{\prime} \frac{-e}{2 \pi} V\left(t^{\prime}\right) .
$$

In analogy with the previous Section, we study the lesser excess spectral function $\Delta \mathcal{A}_{\uparrow}^{<}(\omega)$ for different interaction strengths $K=\{1,0.8,0.6\}$ and charges $Q_{\uparrow}=\{1,1.1\}$. Results are summarized in Fig. 4, where minimal distributions with definite sign are highlighted in red. The first three plots (A, B and C) correspond to the injection of an integer charge $Q_{\uparrow}=1$ and they do not feature divergences for $\omega \rightarrow 0$, in agreement with Eq. (27). In the non-interacting case (plot A), it is known that only Levitons are minimal [20] and the excess spectral function for the square pulse features indeed a negative region. However, the latter becomes less pronounced as the interaction gets stronger (plot B) and it remarkably disappears for strong enough interactions (plot $\mathrm{C}$ ), where the spectral function thus becomes minimal. Let us now also consider the case of non-integer charges. The last three plots (D, E and F) correspond to the case of $Q_{\uparrow}=1.1$ and they therefore feature divergences for $\omega \rightarrow 0$, as predicted by Eq. (27). Here, the interaction strength plays two different roles. On the one hand, as for the Lorentzian pulse, it controls the signs of divergent terms through the coefficients $D_{\uparrow}^{ \pm}$. On the other hand, as for the integer case, it tends to shrink regions which a negative excess spectral function. As a result, provided that the necessary condition (ii) is fulfilled, it is possible to find minimal excess 

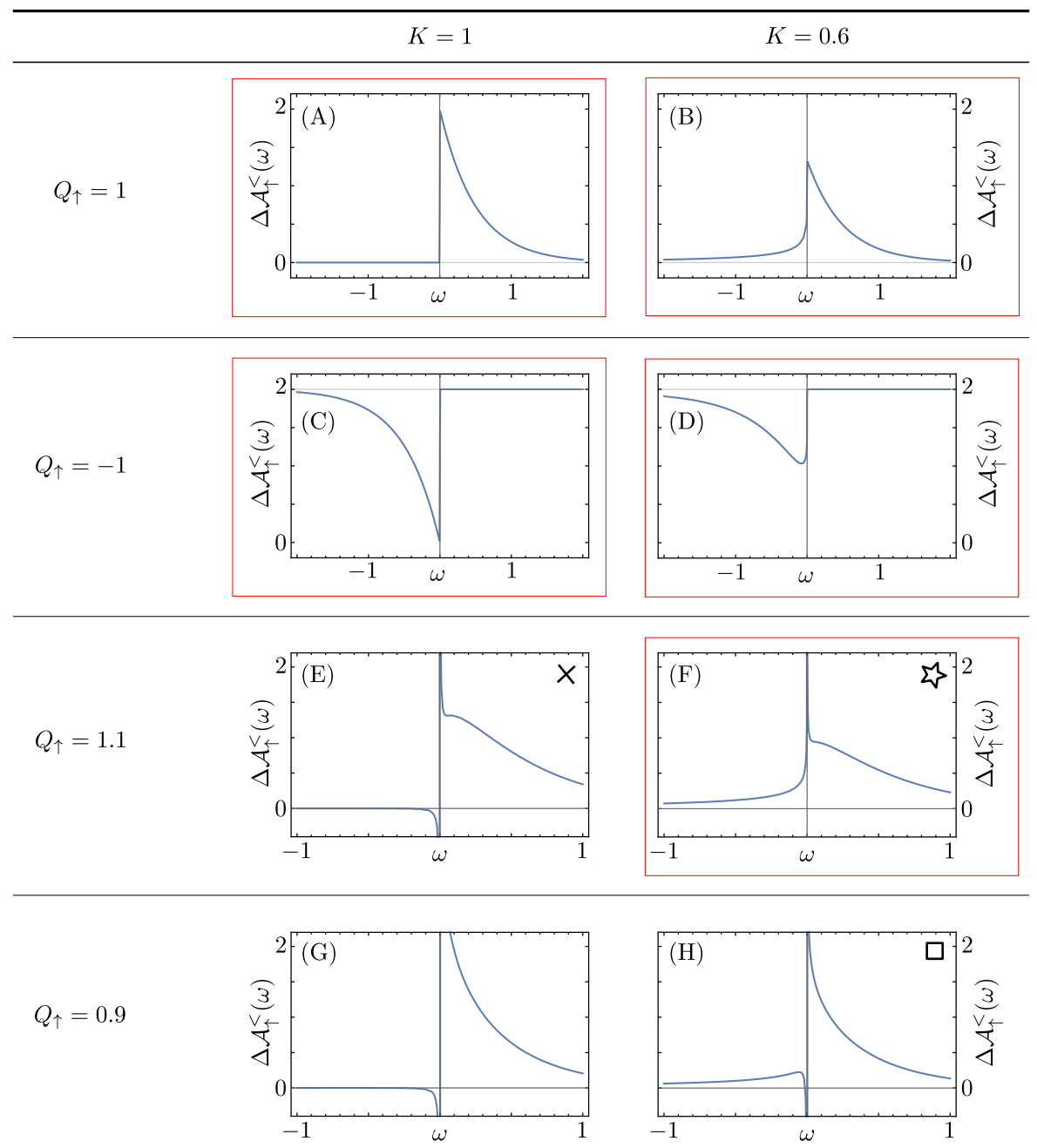

Figure 3. Excess spectral function $\Delta \mathcal{A}_{\uparrow}^{<}$(in units of $w / u$ ), as a function of the energy $\omega$ (in units of $w^{-1}$ ), associated with Lorentzian pulses [see Eq. (30)]. Several different couples of parameters $\left(Q_{\uparrow}, K\right)$ are considered. Plots featuring an excess spectral function with definite sign are highlighted with a red rectangle. The black markers (cross, star, square) are meant to ease the visualization of the corresponding parameters in the $\left(Q_{\uparrow}, K\right)$ plane of Fig. 2 . We choose the cutoff $a=0.01 \mathrm{uw}$.

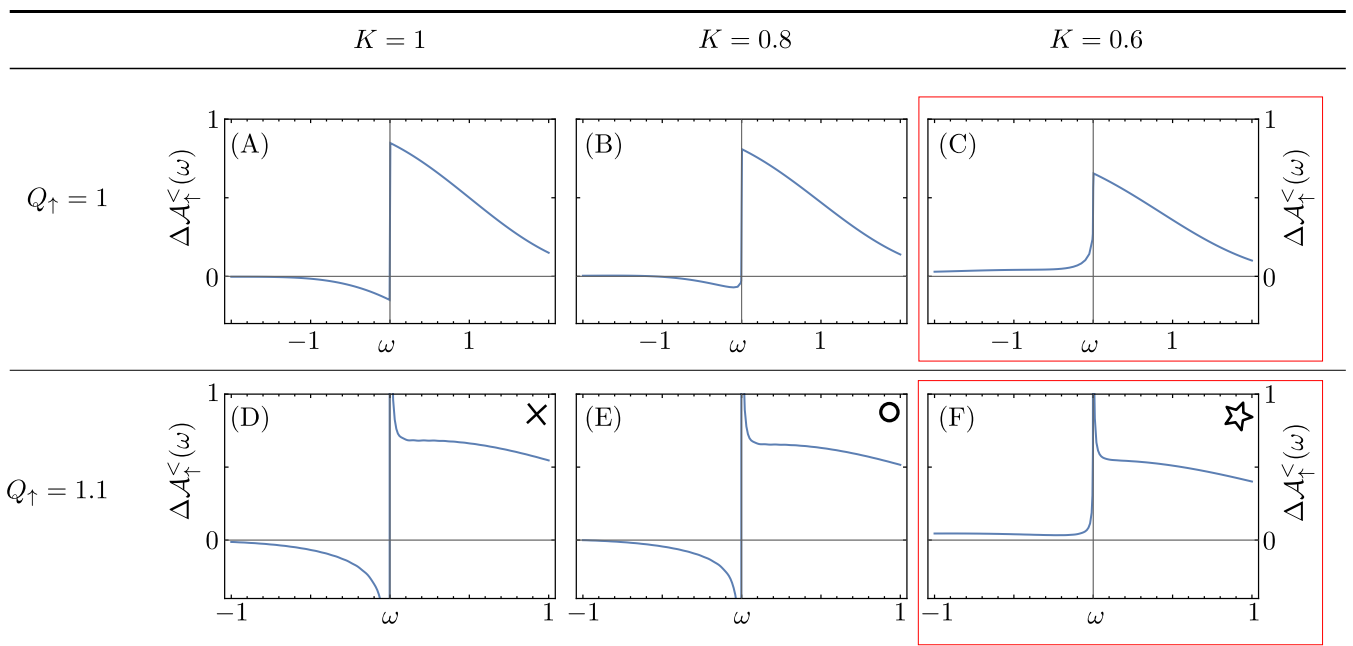

Figure 4. Excess spectral function $\Delta \mathcal{A}_{\uparrow}^{<}$(in units of $w / u$ ), as a function of the energy $\omega$ (in units of $w^{-1}$ ), associated with square pulses [see Eq. (32)]. Several different couples of parameters $\left(Q_{\uparrow}, K\right)$ are considered. Plots featuring an excess spectral function with definite sign are highlighted with a red rectangle. The black markers (cross, circle, star) are meant to ease the visualization of the corresponding parameters in the $\left(Q_{\uparrow}, K\right)$ plane of Fig. 2. We choose the cutoff $a=0.01 u w$. 
spectral function even in the (interacting) non-integer case, as nicely testified by plot $\mathrm{F}$. Therefore, in the presence of interactions, Lorentzian pulses with integer charge are no longer the only drive featuring a minimal spectral function. As an example, we showed that a square pulse, either with integer of non-integer charge, can be associated with a minimal spectral function.

It is important to stress that the peculiarity of quantized Lorentzian pulses surviving even in the presence of interactions emerge when considering another property, namely the noise generated when the excitations created by the voltage pulse are partitioned at a quantum point contact. Indeed, even in the presence of interactions, Lorentzian pulses with integer charge are the only signal producing the minimal noise. As discussed in [87], this property is due to the presence of Heaviside step functions in $\Delta \mathcal{A}_{\uparrow}^{<}(\omega)$ (see, for instance, panel D in Fig. 3) and it is not uniquely related to the concept of minimality meant as an excess spectral function $\Delta \mathcal{A}_{\uparrow}^{<}(\omega)$ with definite sign.

\section{Conclusions}

In this paper, we considered the effects of single voltage pulses applied to a top gate, capacitively coupled with two helical channels. In particular, we studied the excess lesser spectral function of the excitations generated by the pulses. Such a quantity allows indeed to discriminate between minimal excitations, featuring a spectral function with definite sign, and non-minimal ones, featuring sign changes in their spectral functions.

In the non-interacting case, it is well-known that only integer Lorentzian pulses give rise to minimal excitations, which are called Levitons. In the presence of interactions, however, the picture changes dramatically. In the present work, we showed that it is possible to obtain minimal excitations even for non-quantized Lorentzian pulses, which carry non-integer charges, which is not allowed in the absence of interactions. Even more surprisingly, we demonstrated that it is possible to generate minimal excitations even with square pulses, either carrying integer or non-integer charges, provided that the interaction is strong enough. Therefore, the presence of interactions enriches the simple picture valid in non-interacting systems, where Levitons with integer charge are the only kind of excitation exhibiting a minimal spectral function. Quantized Lorentzian pulses maintain a special role when considering the excess noise in the presence of a quantum point contact, as they are the only kind of drive for which it vanishes.

Finally, as discussed in Ref. [87], the described features of the excess spectral functions can be related to local measurements via spin-resolved tunneling spectroscopy.

\section{References}

[1] C. Grenier, R. Hervé, G. Fève, P. Degiovanni, Mod. Phys. Lett. B 25, 1053 (2011)

[2] E. Bocquillon, V. Freulon, F.D. Parmentier, J. Berroir, B. Plaçais, C. Wahl, J. Rech, T. Jonck- heere, T. Martin, C. Grenier et al., Ann. Phys. 526, 1 (2014)

[3] K.v. Klitzing, G. Dorda, M. Pepper, Phys. Rev. Lett. 45, 494 (1980)

[4] A. Stern, Annals of Physics 323, 204 (2008)

[5] M.Z. Hasan, C.L. Kane, Rev. Mod. Phys. 82, 3045 (2010)

[6] X.L. Qi, S.C. Zhang, Rev. Mod. Phys. 83, 1057 (2011)

[7] G. Dolcetto, M. Sassetti, T.L. Schmidt, Riv. Nuovo Cimento 39, 113 (2016)

[8] B.A. Bernevig, Topological insulators and topological superconductors (Cambridge University Press, 2013)

[9] Y. Ren, Z. Qiao, Q. Niu, Reports on Progress in Physics 79, 066501 (2016)

[10] C. Bäuerle, D.C. Glattli, T. Meunier, F. Portier, P. Roche, P. Roulleau, S. Takada, X. Waintal, Reports on Progress in Physics 81, 056503 (2018)

[11] G. Fève, A. Mahé, J.M. Berroir, T. Kontos, B. Plaçais, D.C. Glattli, A. Cavanna, B. Etienne, Y. Jin, Science 316, 1169 (2007)

[12] A. Mahé, F.D. Parmentier, E. Bocquillon, J.M. Berroir, D.C. Glattli, T. Kontos, B. Plaçais, G. Fève, A. Cavanna, Y. Jin, Phys. Rev. B 82, 201309 (2010)

[13] F.D. Parmentier, E. Bocquillon, J.M. Berroir, D.C. Glattli, B. Plaçais, G. Fève, M. Albert, C. Flindt, M. Büttiker, Phys. Rev. B 85, 165438 (2012)

[14] R. Hanbury Brown, R.Q. Twiss, Nature 178, 1046 (1956)

[15] E. Bocquillon, F.D. Parmentier, C. Grenier, J.M. Berroir, P. Degiovanni, D.C. Glattli, B. Plaçais, A. Cavanna, Y. Jin, G. Fève, Phys. Rev. Lett. 108, 196803 (2012)

[16] C.K. Hong, Z.Y. Ou, L. Mandel, Phys. Rev. Lett. 59, 2044 (1987)

[17] E. Bocquillon, V. Freulon, J.M. Berroir, P. Degiovanni, B. Plaçais, A. Cavanna, Y. Jin, G. Fève, Science 339, 1054 (2013)

[18] V. Freulon, A. Marguerite, J.M. Berroir, B. Plaçais, A. Cavanna, Y. Jin, G. Fève, Nature Communications 6, 6854 (2015)

[19] L.S. Levitov, H. Lee, G.B. Lesovik, J. Math. Phys. 37, 4845 (1996)

[20] D.A. Ivanov, H.W. Lee, L.S. Levitov, Phys. Rev. B 56, 6839 (1997)

[21] J. Keeling, I. Klich, L.S. Levitov, Phys. Rev. Lett. 97, 116403 (2006)

[22] J. Dubois, T. Jullien, F. Portier, P. Roche, A. Cavanna, Y. Jin, W. Wegscheider, P. Roulleau, D.C. Glattli, Nature 502, 659 (2013)

[23] J. Dubois, T. Jullien, C. Grenier, P. Degiovanni, P. Roulleau, D.C. Glattli, Phys. Rev. B 88, 085301 (2013)

[24] D. Dasenbrook, C. Flindt, Phys. Rev. B 92, 161412 (2015)

[25] D. Dasenbrook, J. Bowles, J.B. Brask, P.P. Hofer, C. Flindt, N. Brunner, New Journal of Physics 18, 
043036 (2016)

[26] N. Dashti, M. Misiorny, S. Kheradsoud, P. Samuelsson, J. Splettstoesser, Phys. Rev. B 100, 035405 (2019)

[27] N. Dashti, M. Misiorny, P. Samuelsson, J. Splettstoesser, Phys. Rev. Applied 10, 024007 (2018)

[28] J. Rech, D. Ferraro, T. Jonckheere, L. Vannucci, M. Sassetti, T. Martin, Phys. Rev. Lett. 118, 076801 (2017)

[29] L. Vannucci, F. Ronetti, J. Rech, D. Ferraro, T. Jonckheere, T. Martin, M. Sassetti, Phys. Rev. B 95, 245415 (2017)

[30] D. Ferraro, F. Ronetti, J. Rech, T. Jonckheere, M. Sassetti, T. Martin, Phys. Rev. B 97, 155135 (2018)

[31] D. Ferraro, F. Ronetti, L. Vannucci, M. Acciai, J. Rech, T. Jonckheere, T. Martin, M. Sassetti, The European Physical Journal Special Topics 227, 1345 (2018)

[32] D. Ferraro, B. Roussel, C. Cabart, E. Thibierge, G. Fève, C. Grenier, P. Degiovanni, Phys. Rev. Lett. 113, 166403 (2014)

[33] M. Moskalets, Phys. Rev. B 97, 155411 (2018)

[34] M. Moskalets, Phys. Rev. B 91, 195431 (2015)

[35] M. Moskalets, Phys. Rev. Lett. 117, 046801 (2016)

[36] M. Moskalets, G. Haack, Physica E: Lowdimensional Systems and Nanostructures 82, 204 (2016)

[37] M. Moskalets, G. Haack, physica status solidi (b) 254, 1600616 (2017)

[38] F. Ronetti, L. Vannucci, D. Ferraro, T. Jonckheere, J. Rech, T. Martin, M. Sassetti, Phys. Rev. B 98, 075401 (2018)

[39] F. Ronetti, L. Vannucci, D. Ferraro, T. Jonckheere, J. Rech, T. Martin, M. Sassetti, Phys. Rev. B 99, 205406 (2019)

[40] F. Ronetti, M. Acciai, D. Ferraro, J. Rech, T. Jonckheere, T. Martin, M. Sassetti, Entropy 21, 730 (2019)

[41] C. Cabart, B. Roussel, G. Fève, P. Degiovanni, Phys. Rev. B 98, 155302 (2018)

[42] P.P. Hofer, D. Dasenbrook, C. Flindt, physica status solidi (b) 254, 1600582 (2017)

[43] D. Glattli, P. Roulleau, Physica E: Low-dimensional Systems and Nanostructures 76, 216 (2016)

[44] D.C. Glattli, P.S. Roulleau, Phys. Status Solidi (b) 254, 1600650 (2017)

[45] M. Misiorny, G. Fève, J. Splettstoesser, Phys. Rev. B 97, 075426 (2018)

[46] D.C. Glattli, P. Roulleau, Phys. Rev. B 97, 125407 (2018)

[47] M. Acciai, M. Carrega, J. Rech, T. Jonckheere, T. Martin, M. Sassetti, Phys. Rev. B 98, 035426 (2018)

[48] S. Guiducci, M. Carrega, G. Biasiol, L. Sorba, F. Beltram, S. Heun, physica status solidi (RRL) - Rapid Research Letters 13, 1800222 (2019)
[49] M. Kapfer, P. Roulleau, M. Santin, I. Farrer, D.A. Ritchie, D.C. Glattli, Science 363, 846 (2019)

[50] D. Ferraro, J. Rech, T. Jonckheere, T. Martin, Phys. Rev. B 91, 075406 (2015)

[51] M. Acciai, F. Ronetti, D. Ferraro, J. Rech, T. Jonckheere, M. Sassetti, T. Martin, Phys. Rev. B 100, 085418 (2019)

[52] P.P. Hofer, M. Büttiker, Phys. Rev. B 88, 241308 (2013)

[53] A. Inhofer, D. Bercioux, Phys. Rev. B 88, 235412 (2013)

[54] D. Ferraro, C. Wahl, J. Rech, T. Jonckheere, T. Martin, Phys. Rev. B 89, 075407 (2014)

[55] A. Calzona, M. Acciai, M. Carrega, F. Cavaliere, M. Sassetti, Phys. Rev. B 94, 035404 (2016)

[56] F. Dolcini, R.C. Iotti, A. Montorsi, F. Rossi, Phys. Rev. B 94, 165412 (2016)

[57] F. Dolcini, Phys. Rev. B 95, 085434 (2017)

[58] F. Dolcini, F. Rossi, The European Physical Journal Special Topics 227, 1323 (2018)

[59] F. Ronetti, M. Carrega, M. Sassetti, arXiv:1911.03697 (2019)

[60] A. Blasi, A. Braggio, M. Carrega, D. Ferraro, N. Maggiore, N. Magnoli, New Journal of Physics 14, 013060 (2012)

[61] J. Strunz, J. Wiedenmann, C. Fleckenstein, L. Lunczer, W. Beugeling, V.L. Müller, P. Shekhar, N.T. Ziani, S. Shamim, J. Kleinlein et al., Nature Physics (2019)

[62] C. Grenier, J. Dubois, T. Jullien, P. Roulleau, D.C. Glattli, P. Degiovanni, Phys. Rev. B 88, 085302 (2013)

[63] C. Wahl, J. Rech, T. Jonckheere, T. Martin, Phys. Rev. Lett. 112, 046802 (2014)

[64] M. Hashisaka, N. Hiyama, T. Akiho, K. Muraki, T. Fujisawa, Nature Physics 13, 559 (2017)

[65] M. Hashisaka, T. Fujisawa, Reviews in Physics 3, 32 (2018)

[66] L. Bellentani, P. Bordone, X. Oriols, A. Bertoni, Phys. Rev. B 99, 245415 (2019)

[67] D. Ferraro, M. Carrega, A. Braggio, M. Sassetti, New Journal of Physics 16, 043018 (2014)

[68] A. Braggio, D. Ferraro, M. Carrega, N. Magnoli, M. Sassetti, New Journal of Physics 14, 093032 (2012)

[69] K.V. Pham, M. Gabay, P. Lederer, Phys. Rev. B 61, 16397 (2000)

[70] I. Safi, H.J. Schulz, Phys. Rev. B 52, R17040 (1995)

[71] K.L. Hur, B.I. Halperin, A. Yacoby, Annals of Physics 323, 3037 (2008)

[72] H. Kamata, N. Kumada, M. Hashisaka, K. Muraki, T. Fujisawa, Nature Nanotechnology 9, 177 (2014)

[73] E. Perfetto, G. Stefanucci, H. Kamata, T. Fujisawa, Phys. Rev. B 89, 201413 (2014)

[74] A. Calzona, M. Carrega, G. Dolcetto, M. Sassetti, Phys. Rev. B 92, 195414 (2015) 
[75] A. Calzona, M. Carrega, G. Dolcetto, M. Sassetti, Physica E Low-Dimensional Systems and Nanostructures 74, 630 (2015)

[76] A. Calzona, F.M. Gambetta, M. Carrega, F. Cavaliere, M. Sassetti, Phys. Rev. B 95, 085101 (2017)

[77] P. Brasseur, N.H. Tu, Y. Sekine, K. Muraki, M. Hashisaka, T. Fujisawa, N. Kumada, Phys. Rev. B 96, 081101 (2017)

[78] Y. Jompol, C.J.B. Ford, J.P. Griffiths, I. Farrer, G.A.C. Jones, D. Anderson, D.A. Ritchie, T.W. Silk, A.J. Schofield, Science 325, 597 (2009)

[79] O.M. Auslaender, H. Steinberg, A. Yacoby, Y. Tserkovnyak, B.I. Halperin, K.W. Baldwin, L.N. Pfeiffer, K.W. West, Science 308, 88 (2005)

[80] R. Stühler, F. Reis, T. Müller, T. Helbig, T. Schwemmer, R. Thomale, J. Schäfer, R. Claessen, Nature Physics (2019)

[81] T. Li, P. Wang, H. Fu, L. Du, K.A. Schreiber, X. Mu, X. Liu, G. Sullivan, G.A. Csáthy, X. Lin et al., Phys. Rev. Lett. 115, 136804 (2015)

[82] J.I. Väyrynen, F. Geissler, L.I. Glazman, Phys. Rev. B 93, 241301 (2016)

[83] J. Voit, Reports on Progress in Physics 58, 977 (1995)

[84] T. Giamarchi, Quantum Physics in One Dimension (Oxford University Press, 2003)

[85] F.D.M. Haldane, Phys. Rev. Lett. 47, 1840 (1981)

[86] F. Geissler, F. Crépin, B. Trauzettel, Phys. Rev. B 92, 235108 (2015)
[87] M. Acciai, A. Calzona, M. Carrega, T. Martin, M. Sassetti, New Journal of Physics 21, 103031 (2019)

[88] A. Calzona, F.M. Gambetta, F. Cavaliere, M. Carrega, M. Sassetti, Phys. Rev. B 96, 085423 (2017)

[89] A. Calzona, F.M. Gambetta, M. Carrega, F. Cavaliere, T.L. Schmidt, M. Sassetti, SciPost Phys. 4, 23 (2018)

[90] J. Tersoff, D.R. Hamann, Phys. Rev. B 31, 805 (1985)

[91] C.J. Chen, Introduction to Scanning Tunneling Microscopy: Second Edition (Oxford University Press, 2007)

[92] In order to preserve gauge invariance of the Green function, an extra phase term, called Wilson line, is in general needed in the definition (16), as discussed more in detail in Refs. [56, 58]. However, with our choice of the external drive in Eq. (3), this term contributes only at $x<0$ and it is thus irrelevant to our discussion, which focuses on $x>0$.

[93] M. Acciai, A. Calzona, G. Dolcetto, T.L. Schmidt, M. Sassetti, Phys. Rev. B 96, 075144 (2017)

[94] P.W. Anderson, Phys. Rev. Lett. 18, 1049 (1967)

[95] G.D. Mahan, Many-Particle Physics (Plenum, 1981)

[96] H.W. Lee, L.S. Levitov, arXiv:cond-mat/9312013 (1993)

[97] M. Vanević, Y.V. Nazarov, W. Belzig, Phys. Rev. Lett. 99, 076601 (2007) 